\title{
Analysis of Five Pharmacologically Active Compounds from Rhodiola for Natural Product Drug Discovery with Capillary Electrophoresis
}

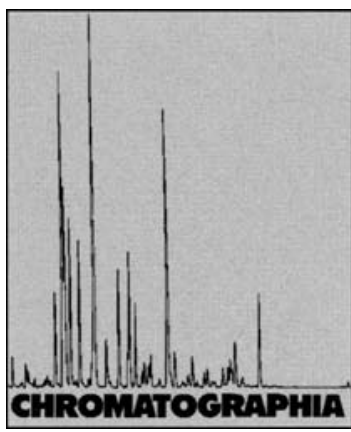

2004, 60, 589-595

\author{
Yourui Suo ${ }^{1, \bowtie}$, Honglun Wang ${ }^{1,4}$, Yulin Li ${ }^{1,4}$, Jinmao You ${ }^{2}$, Hanqing Wang ${ }^{3}$ \\ ${ }_{1}^{1}$ Northwest Plateau Institute of Biology, Chinese Academy of Sciences, Xining, 810008, P.R. China; E-Mail: yrsuo@mail.nwipb.ac.cn \\ ${ }^{2}$ Department of Chemistry, Qufu Normal University, Qufu, 273165, P.R. China \\ ${ }^{3}$ Lanzhou Institute of Chemical Physics, Chinese Academy of Sciences, Lanzhou, 730000, P.R. China \\ ${ }^{4}$ Graduate School of the Chinese Academy of Sciences, Beiing, 100039, P.R. China
}

Received: 15 April 2004 / Revised: 23 July 2004 / Accepted: 2 August 2004

Online publication: 18 October 2004

\begin{abstract}
A rapid capillary electrophoresis method for the separation of five natural pharmacologically active compounds from extracted Rhodiola, namely salidroside, tyrosol, rhodionin, gallic acid and ethyl gallate has been developed. The separation of five natural pharmacologically active compounds was carried out in a fused-silica capillary with $14 \mathrm{mM}$ boric acid, $30 \mathrm{mM}$ SDS and $2.5 \%$ acetonitrile, adjusted to $\mathrm{pH} 10.7$ with $\mathrm{NaOH}$. Applied potential was $21 \mathrm{kV}$. The temperature of the capillary was maintained at $25{ }^{\circ} \mathrm{C}$ by the instrument thermostating system, with the correlation coefficients of $0.9805-0.9989$ for migration time, and relative standards of $<3.52 \%$ for peak areas. The established method is rapid and reproducible for the separation of five natural pharmacologically compounds from extracts of Rhodiola with satisfactory results.
\end{abstract}

\section{Keywords}

Capillary electrophoresis

Pharmacologically active compounds

Rhodiola crenucata

Rhodiola quadrifida

\section{Introduction}

Within the pharmaceutical industry, the identification of novel active compounds through the use of powerful tools can be considered to be one of the most important discoveries for natural drug. Samples isolated for these compounds often are initially generated as complex mixtures consisting of multiple components, and powerful analysis tools are required for both the separation and characterization of the compounds in these mixtures in a rapid manner. Specifically, the intrinsic complexity of nat- ural product extracts represents both a source of extremely high compound diversity and a significant separation/ deconvolution challenge. Traditionally, reversed-phase liquid chromatography has served as an effective technique for the separation of many of the components in complex mixtures that are under evaluation for drug discovery. Reversedphase column packing has been successfully utilized in conjunction with volatile mobile phases to provide rapid high-throughput characterization of samples via liquid chromatography-electrospray ionization mass spectrometry
(LC-ESI-MS). Normal-phase liquid chromatography (NPLC) has historically been employed for separations using non-aqueous mobile phases such as hexane or chloroform together with silica or alumina stationary phases. However, the application of NPLC to the separation of samples of biological origin has been extremely limited because of the problems associated with dissolving hydrophilic materials in non-aqueous mobile phases. Another difficulty associated with the use of NPLC for complex mixture characterization is the fact that several commonly used organic solvents such as benzene and toluene are not compatible with the electrospray process now prevalently utilized for molecular mass determinations. NPLC using mobile phases containing a low percentage of an aqueous component has been employed, but the chromatography obtained in these systems has been of notoriously poor reproducibility. An alternative to NPLC for the chromatography of natural product extracts is hydrophilic interaction chromatography (HILIC). HILIC is similar to NPLC in that elution is promoted by the use of polar mobile phases, but is unique in that the presence of water in the mobile phase is crucial for the establishment of a stagnant enriched aqueous layer on the surface of the stationary phase into which analytes may selectively partition, as described by Alpert [1]. The HILIC separations of carbohydrates [2-4] and proteins [5-7] have been reported, together with some examples of pre- 


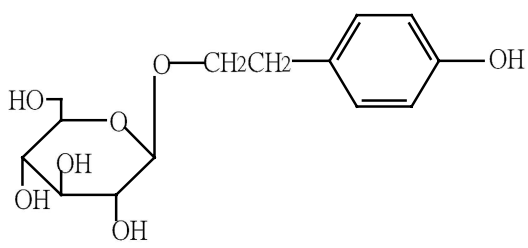

Salidroside

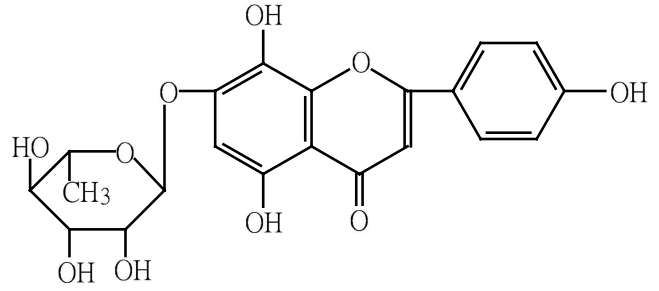

Rhodionin

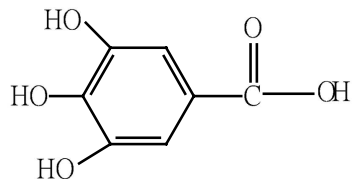

Gallic acid

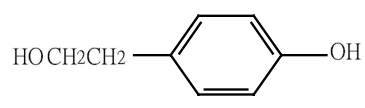

Tyrosol

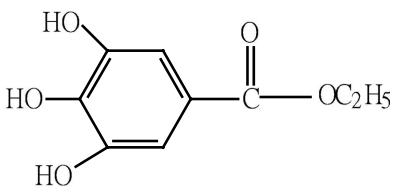

Ethyl gallate

Fig. 1. Structure of the natural product test standards

liminary evaluations of the LC-MS technique.

For the drug discovery efforts currently taking place within the pharmaceutical industry, natural product extracts have been found to provide a valuable source of molecular diversity which is complementary to that provided by traditional synthetic organic methods or combinatorial chemistry. However, there exists a need for analytical tools that can facilitate the separation and characterization of components from these sources in a rapid manner. Traditional chromatography has served as an effective technique for the separation of many of the components in complex mixtures. However, a great quantity of organic solvent must be employed with drastic toxicity such as methanol, $n$-hexane and benzene. In addition, the use of LC is suffered from its column being easily contaminated for silica or alumina stationary phases with hardly regeneration. Compared with LC, capillary electrophoresis (CE) is a conceptually simple technique that offers highly efficient separations with the requirement of only small amounts of samples. CE is also an effective tool for the separation of polar natural compounds with high efficiency, short analysis time, low consumption, and multiple modes to be chosen such as CZE, CEC, MEKC, CGE, CITP and capillary isoeletric focus.

Although numerous publications were reported for the analysis of effective components from different traditional Chinese medicines (TCM) [8-12], the further composition analysis from TCM plants can be considered to be one of the most important discoveries in order to discover natural drug. Rhodiola is a traditional Chinese medicine belonging to the family of Crassulaceae that mainly distributes in north atmosphere with about 90 species recorded in the world and above 70 species found in China [13]. The extracts of Rhodiola exhibits different efficacy from clinical examination such as exciting the central nervous system, anti-virus, anti-lacking of oxygen, anti-cold and anti-tatigue [14-18]. The separation of several pure compounds from Rhodiola plants by column chromatography was described [19-21]. However, the employment of $\mathrm{CE}$ as a tool for the simultaneous sep- aration five natural polar compounds from Rhodiola for drug discovery has not yet been investigated or exploited. In this study, the influence of parameters upon the retention and resolution of polar natural product compounds (Fig. 1) was evaluated with the goal of optimization the utility of CE.

\section{Experimental}

\section{Instrumentation}

Experiments were performed on HP-3D CE system with a diode-array detector (Agilent Technologies, USA). Data were collected on a PC computer using HP $3 \mathrm{D}$ ChemStation. In all experiments, $48.5 \mathrm{~cm} \times 50 \mu \mathrm{m}(40 \mathrm{~cm}$ to the detector $)$ fused silica capillaries (Yongnian Optical Fiber Factory, Hebei, P. R. China) were used. The samples were detected at 221 $\mathrm{nm}$. Before use, new capillaries were flushed with $1.0 \mathrm{M} \mathrm{NaOH}$ for $1 \mathrm{~h}$ then flush with re-distilled water and background buffer for $10 \mathrm{~min}$. Between runs the capillary was rinsed under pressure with $0.1 \mathrm{M} \mathrm{NaOH}$, re-distilled water, and running buffer for $2 \mathrm{~min}$ each. The temperature was maintained at $25^{\circ} \mathrm{C}$. The other conditions are as follows: applied voltage $21 \mathrm{KV}$, samples were injected by applying a pressure of $50 \mathrm{mbar}$ for $4 \mathrm{~s}$.

\section{Chemicals}

Potassium hydroxide was purchased from Jining Chemical Reagent Co (Shandong, China). HPLC grade of methanol, acetonitrile were obtained from Shanghai Chemical Reagent Co (Shanghai, China). Water was purified on a Milli-Q system (Millipore, Bedford, MA,USA). All other reagents used in this study were also of analytical grade unless otherwise stated. The five standard samples: salidroside, tyrosol, rhodionin and gallic acid were isolated from Rhodiola crenulata (HK.f.et.thoms)H.Ohha, and ethyl gallate from Rhodiola quadrifida. Their structures were confirmed by comparing their melting points, ${ }^{1} \mathrm{H}-\mathrm{NMR}$, IR, UV and MS data with those given in the literatures [19-20]. 


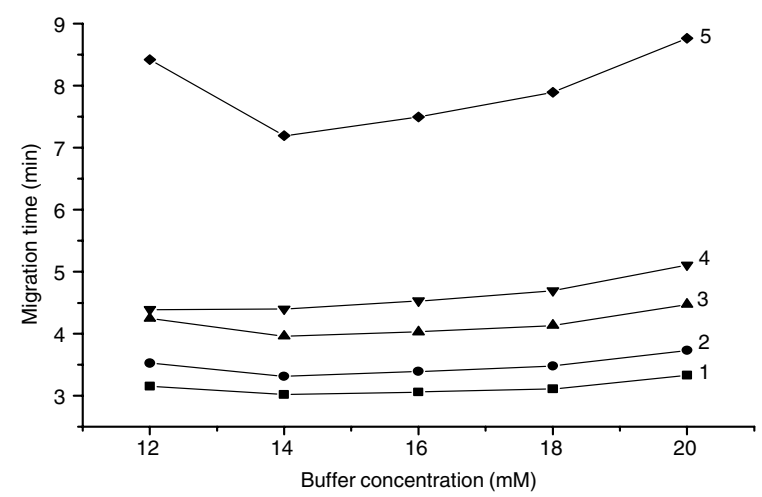

Fig. 2. Effect of buffer concentrations on the separation of five compounds. Conditions: borate buffer at pH 10.7 containing $30 \mathrm{mM}$ SDS and $2.5 \%$ acetonitrile; applied voltage, $21 \mathrm{kV}$; temperature, $25^{\circ} \mathrm{C}$; detection at $221 \mathrm{~nm}$

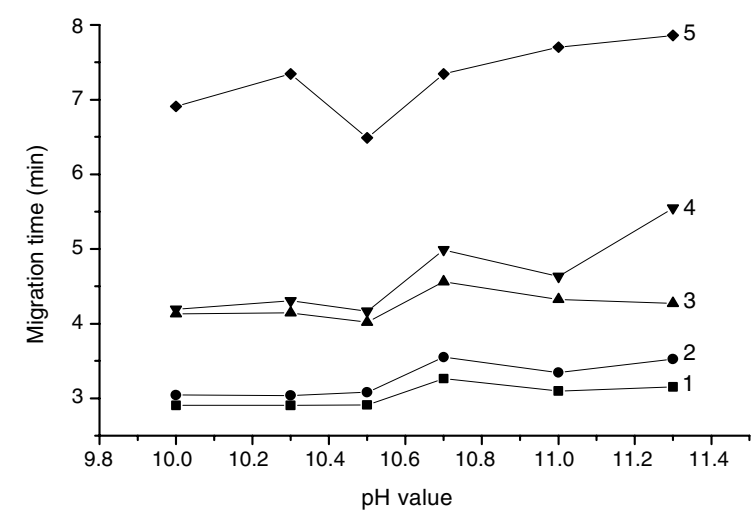

Fig. 3. Effect of $\mathrm{pH}$ on the separation of five compounds. Conditions: borate buffer was $14 \mathrm{mM}$ borate, other conditions as in Fig. 2

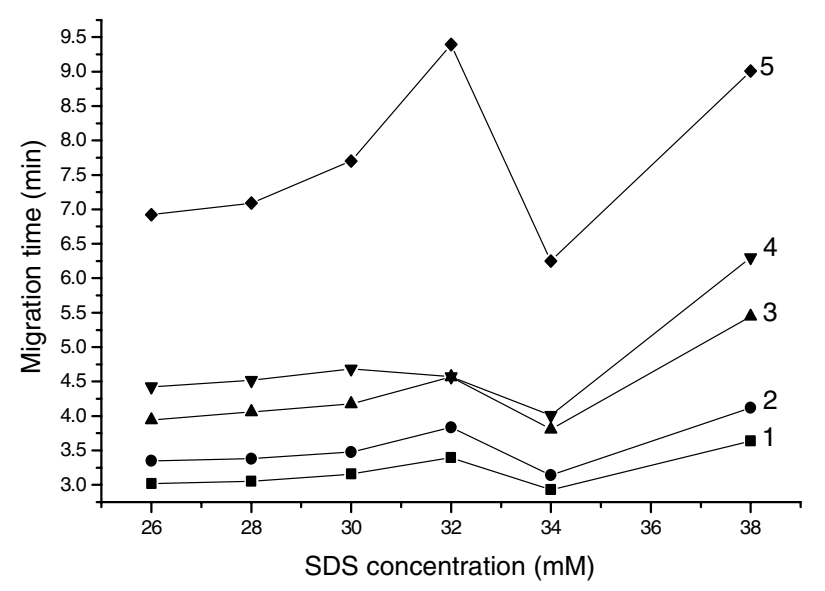

Fig. 4. Effect of SDS concentration on the separation of five compounds. Conditions: borate buffer was $14 \mathrm{mM}$, borate at $\mathrm{pH}$ 10.7, other conditions as Fig. 2

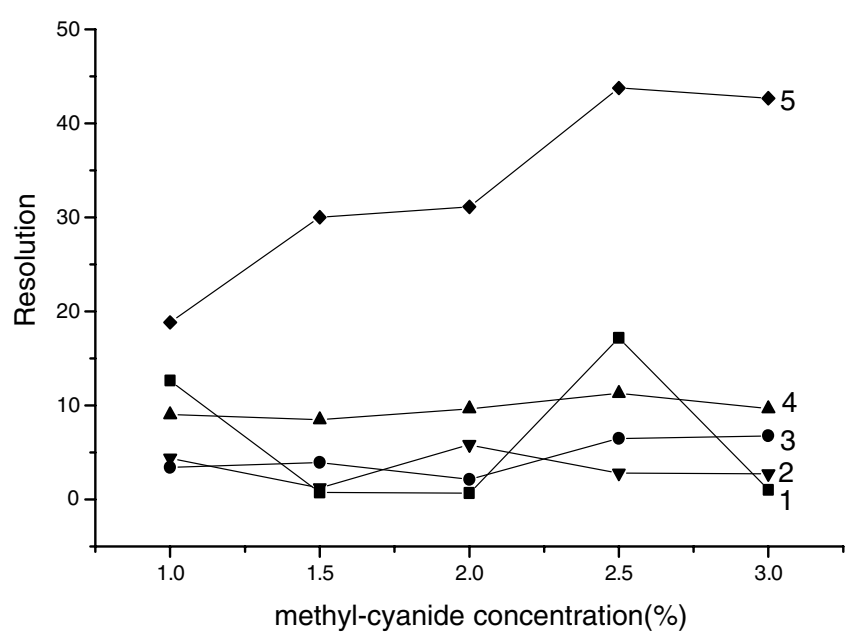

Fig. 5. Effect of acetonitrile concentration on the separation of five compounds. Conditions: $30 \mathrm{mM}$ SDS, other conditions as Fig. 4

\section{Preparation of Standard Solutions}

The standard solutions of five polar components $\left(1.0 \times 10^{-3} \mathrm{M}\right)$ were prepared by dissolving corresponding amounts of pure compounds in $10 \mathrm{~mL}$ of $50 \%$ acetonitrile. Corresponding low concentration of solution $\left(1.0 \times 10^{-5} \mathrm{M}\right)$ was obtained by diluting the stock solution with acetonitrile. When not in use, all reagent solutions were stored at $4{ }^{\circ} \mathrm{C}$ in a refrigerator or at $-20{ }^{\circ} \mathrm{C}$ in a refrigerator until $\mathrm{CE}$ analysis.

Rhodiola was collected from QinghaiTibet Plateau and washed successively with $20 \mathrm{~mL}$ each of water and deionized water. The washed Rhodiola was dried under a stream of nitrogen and broken to powdered samples. To a $10 \mathrm{~mL}$ of roundbottom flask, a $1.0 \mathrm{~g}$ powdered Rhodiola and $10 \mathrm{~mL} 80 \%$ methanol was added. The contents of the flask were allowed to incubate at room temperature for $24 \mathrm{~h}$, then immersed in a sonicator water bath and the sample was sonicated in $5 \mathrm{~min}$ intervals for $30 \mathrm{~min}$. The contents were then centrifuged at a speed of $4000 \mathrm{rpm}$ for $15 \mathrm{~min}$. The supernatant was collected and stored at $4{ }^{\circ} \mathrm{C}$ in a refrigerator until $\mathrm{CE}$ analysis.

\section{Results and Discussion}

The main challenge of the present work was to test the feasibility for the separation of five natural pharmacologically active compounds from extracted Rhodiola with CE in a variety of conditions including migration time, temperature, $\mathrm{pH}$ of buffers and solvents.

\section{Effect of Buffer Concentrations}

Several types of buffers were tested in this study for separation of five polar compounds, including carbonate buffers, phosphate buffers and borate buffers. The results indicated that a reasonably good separation of all five compounds was obtained with borate buffers at concentrations of $14-20 \mathrm{mM}$ with $\mathrm{pH}$ 11. The result is shown in Fig. 2. As can be seen from Fig. 2, with borate buffer concentration $<12$, tyrosol and rhodionin were co-eluted. To achieve optimal separation, operation at buffer concentrations at $14-20 \mathrm{mM}$ resulted in obviously baseline resolution. Interestingly, migration times did no obvious increase for polar compounds with only an exception being gallic acid (from 7.45 to $9.0 \mathrm{~min}$ ). With high buffer concen- 

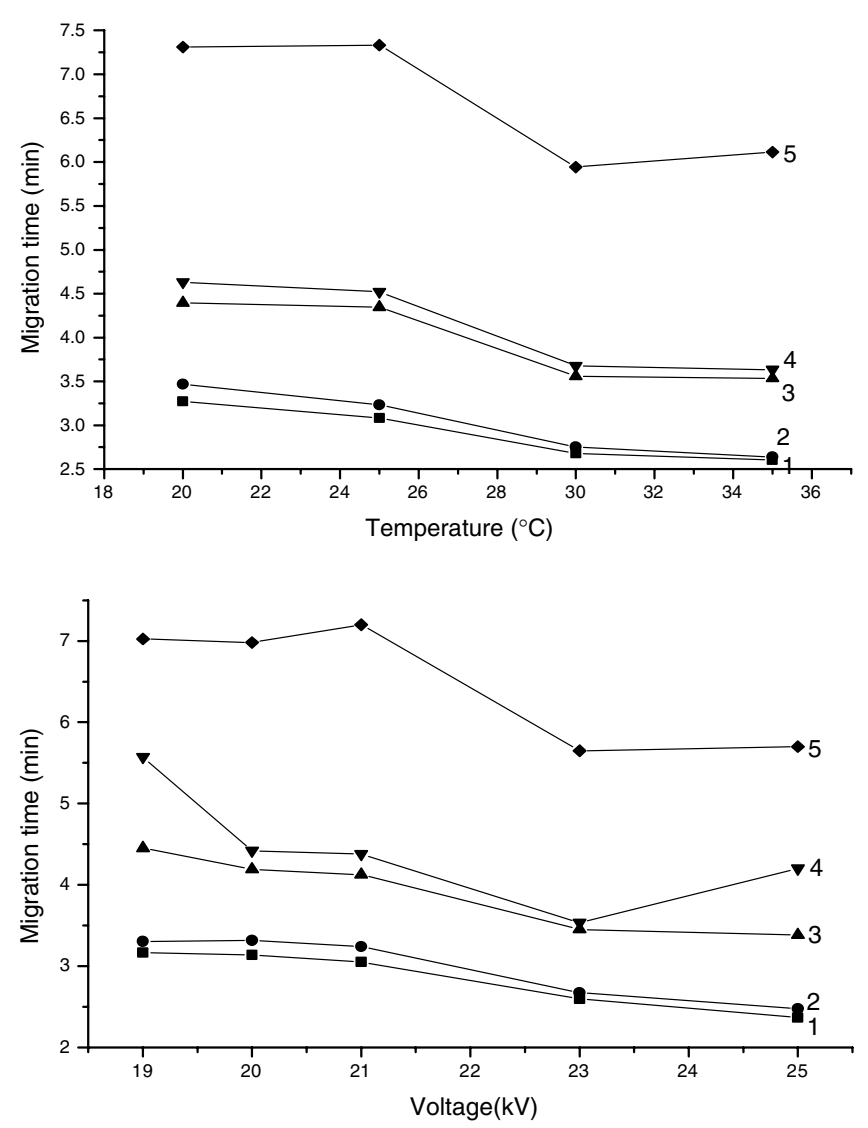

Fig. 6. Effects of capillary temperature and applied voltage on the migration times of five compounds. Conditions: $2.5 \%$ acetonitrile, other conditions as Fig. 5. In the top panel is represented the effect of capillary temperature, in the bottom is represented the effect of voltage

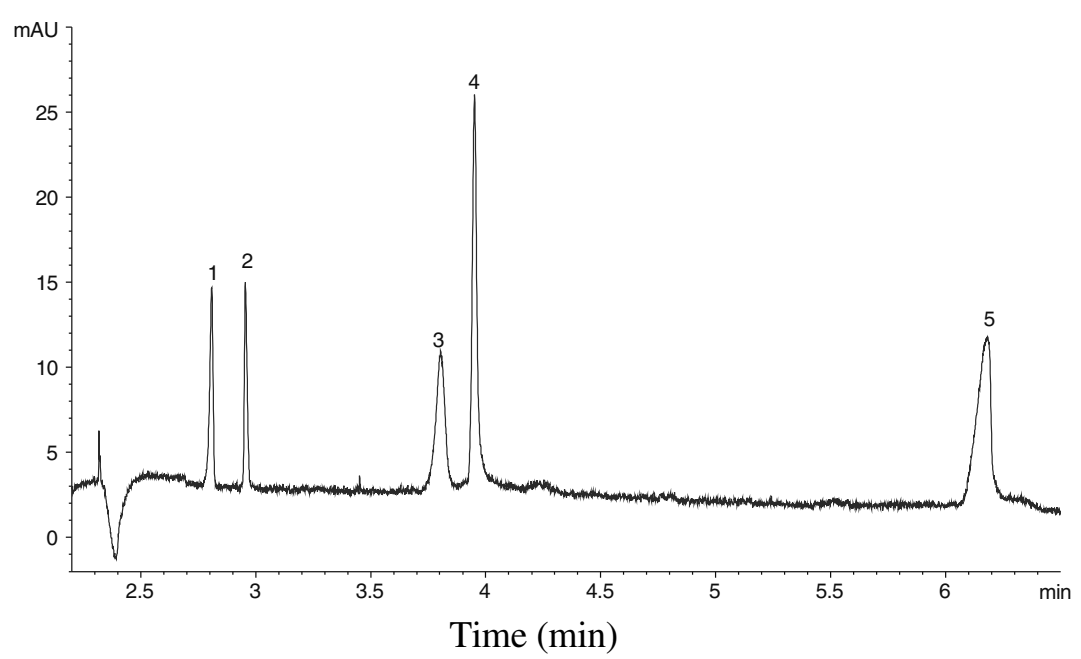

Fig. 7. Electropherogram of standard solution of five polar natural products. Running buffer: $14 \mathrm{mM}$ broate buffer containing $30 \mathrm{mM}$ SDS and $2.5 \%$ acetonitrile, other conditions and peak identification as Fig. 2

trations of $>14 \mathrm{mM}$, an increase ionic strength will result in lower EOF. Taking both the shorter run-time and higher zeta potential into consideration, the borate concentration was selected at $14 \mathrm{mM}$.

\section{Effect of $\mathbf{p H}$}

The effect of $\mathrm{pH}$ on the resolution was investigated with borate buffer in the $\mathrm{pH}$ range of 10.0-11.5. As shown in the Fig. 3, the resolution of five polar com- pounds in CE running procedure increase progressively with increasing $\mathrm{pH}$ of buffers. But outside this range, particularly in $\mathrm{pH}<10.0$, salidroside and tyrosol were co-eluted. At the same time, tyrosol and rhodionin were partially co-eluted. To achieve optimal derivatization, the $\mathrm{pH}$ should be as high as possible. With $\mathrm{pH}>10.7$, a complete baseline resolution of five polar compounds was observed. At higher $\mathrm{pH}$ values $(>10.7)$, a further better the resolution was obtained. However, the hydrolysis of ethyl gallate is favored. Particularity, above $\mathrm{pH} 11.0$, hydrolysis of ethyl gallate became significant and lowered the apparent analytical concentration in real samples. In addition, the running time was prolonged at higher $\mathrm{pH}$ buffers. Therefore, a $0.2 \mathrm{M}$ borate buffer solution at $\mathrm{pH} 10.7$ was used in the final optimization.

\section{Effect of SDS Concentration}

The effect of SDS concentrations on the resolution was investigated for five polar compounds. For this experiment, Six CE running solutions including 26-38 $\mathrm{mM}$ SDS were, respectively, prepared with the optimization $\mathrm{pH}$ and buffers as described above. The effects of SDS on resolution of compounds are shown in Fig. 4. As can be seen, the migration time and corresponding resolution of solutes increases with increasing SDS concentrations. Worthy of noting, tyrosol and rhodionin were partially co-eluted when SDS were in the concentrations range of 32-34 mM. Although further better resolution could be obtained with $38 \mathrm{mM}$ SDS concentration, the running time was also increased by $25 \%$ compared to the $30 \mathrm{mM}$ SDS. Therefore, $30 \mathrm{mM}$ SDS was selected as the optimal SDS concentration since this provided the shortest analysis time, and still gave good separation of all five polar compounds.

\section{Effect of Organic Modifier}

An increase in the acetonitrile concentration increased the flow-rate, partially because the ionic strength was decreased and partially because a greater amount of acetonitrile increases the EOF[22]. The increase in EOF observed at higher concentration of acetonitrile is believed to be caused by changes in the viscosity 
and the zeta potential [23]. Increasing the amount of acetonitrile in the background electrolyte (BE) also made the electrolyte more polar, and this changed the partitioning equilibrium, and thereby affected the selectivity and resolution and decreased the retention of the analytes. The effect of SDS concentrations on the resolution was investigated for five polar compounds. For this experiment, $1.0 \%-3.0 \%$ of acetonitrile solutions were investigated for optimal separation of all five polar compounds. Fig. 5 shows the effect of acetonitrile concentration on the resolution. When the acetonitrile concentration was increased to $2.5 \%$, this separation was improved and complete baseline separation was achieved. For the final method, $2.5 \%$ of acetonitrile in the eluent was chosen.

\section{Effect of Temperature and Voltage}

The separation selectivity was affected slightly by capillary temperature and the applied voltage. As the temperature was increased from 20 to $35^{\circ} \mathrm{C}$. The viscosity was reduced and effect the distribution constants of solutes, and this will result in decreasing retention (peaks migration time was shorten (Fig. 6a). A complete baseline resolution is still observed. To decrease Joul heating, $25^{\circ} \mathrm{C}$ was used for the separation of solutes. The applied voltage had a large effect than capillary temperature on the migration time (Fig. 6b). Taking both short migration time and the good resolution into consideration, the applied voltage was selected $21 \mathrm{kV}$. As a result of these experiments, the optimum separation conditions were set at $30 \mathrm{mM}$ SDS, $2.5 \%(V / V)$ acetonitrile containing $14 \mathrm{mM}$ borate buffer at $25^{\circ} \mathrm{C}$ with $21 \mathrm{kV}$ voltage. Fig. 7 shows the electropherogram of the five standard polar compounds under the proposed conditions.

\section{Linearity and Reproducibility}

Repeatability was determined by carrying out six successive injections of standard solution within one day. The relative standard deviation (RSD) of migration times and peak areas are lis-
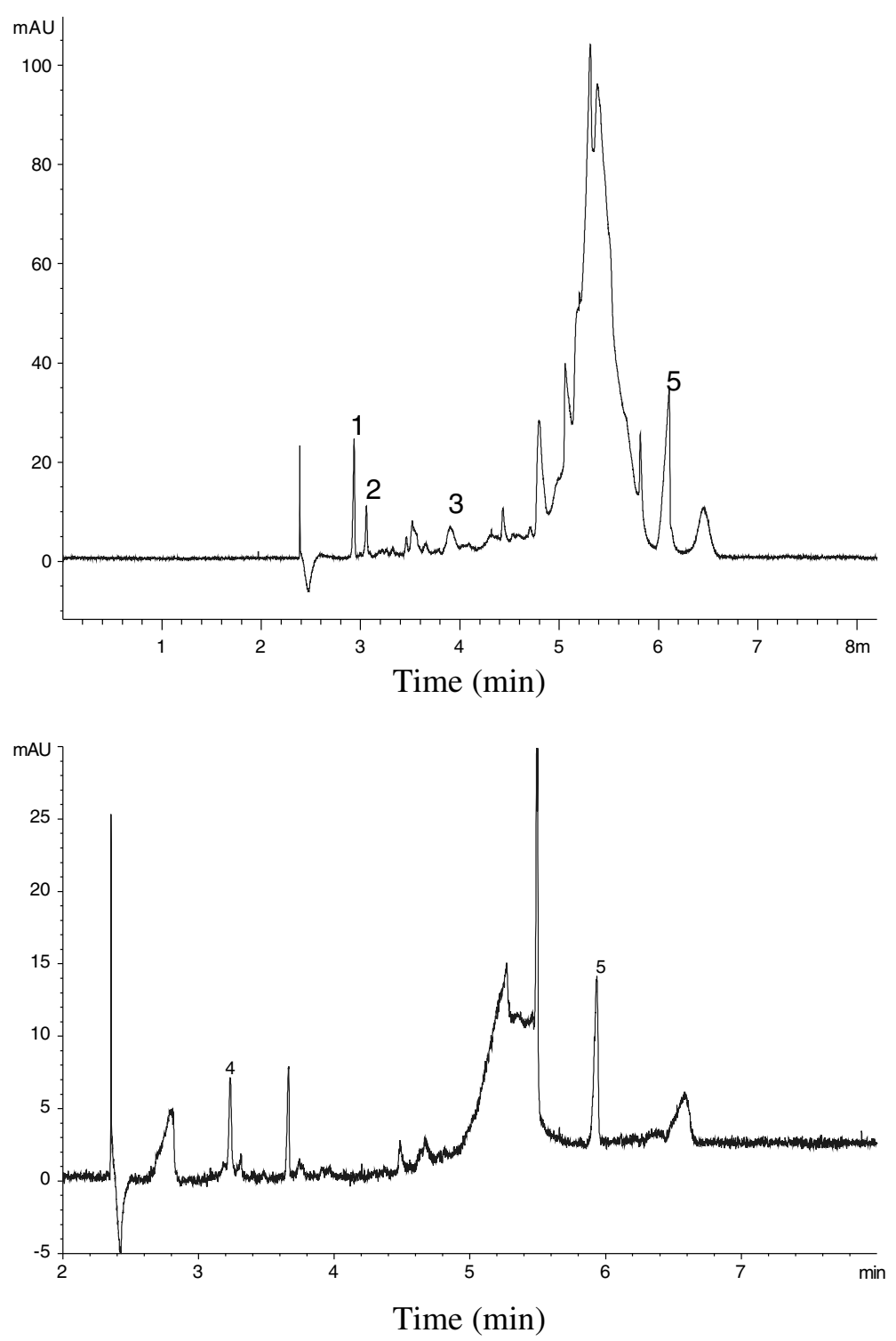

Fig. 8. Electropherogram of extracts of Rhodiola plants using methanol as solvent. Separation conditions as Fig. 7. In the top panel is represented the Rhodiola crenulata; in the bottom is represented the Rhodiola quadrifida

ted in Table 1. The linear relationships between the concentration of analytes and the corresponding peak area are listed in Table 2. The data show that the explored approach is suitable for quantification.

\section{Separation of Extracted Sample}

Extracted methanol solutions from several Rhodiola plants were directly injected into the capillary. Separation of the five compounds was achieved within $7 \mathrm{~min}$ (Fig. 8). The peaks were identified with spiking standards. The on-line UV spec- tra of compounds in Rhodiola (right) well matched the five standard samples (left) (Fig. 9). The contents of salidroside, tyrosol, rhodionin and gallic acid from extracted Rhodiola crenulata were $0.419 \%, 0.086 \%, 0.130 \%$ and $0.184 \%$ $(\mathrm{mg} / \mathrm{mg})$, respectively. The recovery was determined by addition of a known amount of standards into the methanol extracts. The results were $102.1 \%$ for salidroside D-glucopyanoside, $96.4 \%$ for tyrosol, ethanol, $97.8 \%$ for rhodionin and $104.2 \%$ for 3,4,5-trihydroxybenzoic acid, respectively. The contents of ethyl gallate and gallic acid in Rhodiola quadrifida were $0.064 \%$ and $0.071 \%$ with the recoveries $98.3 \%$ and $96.2 \%$, respectively. 

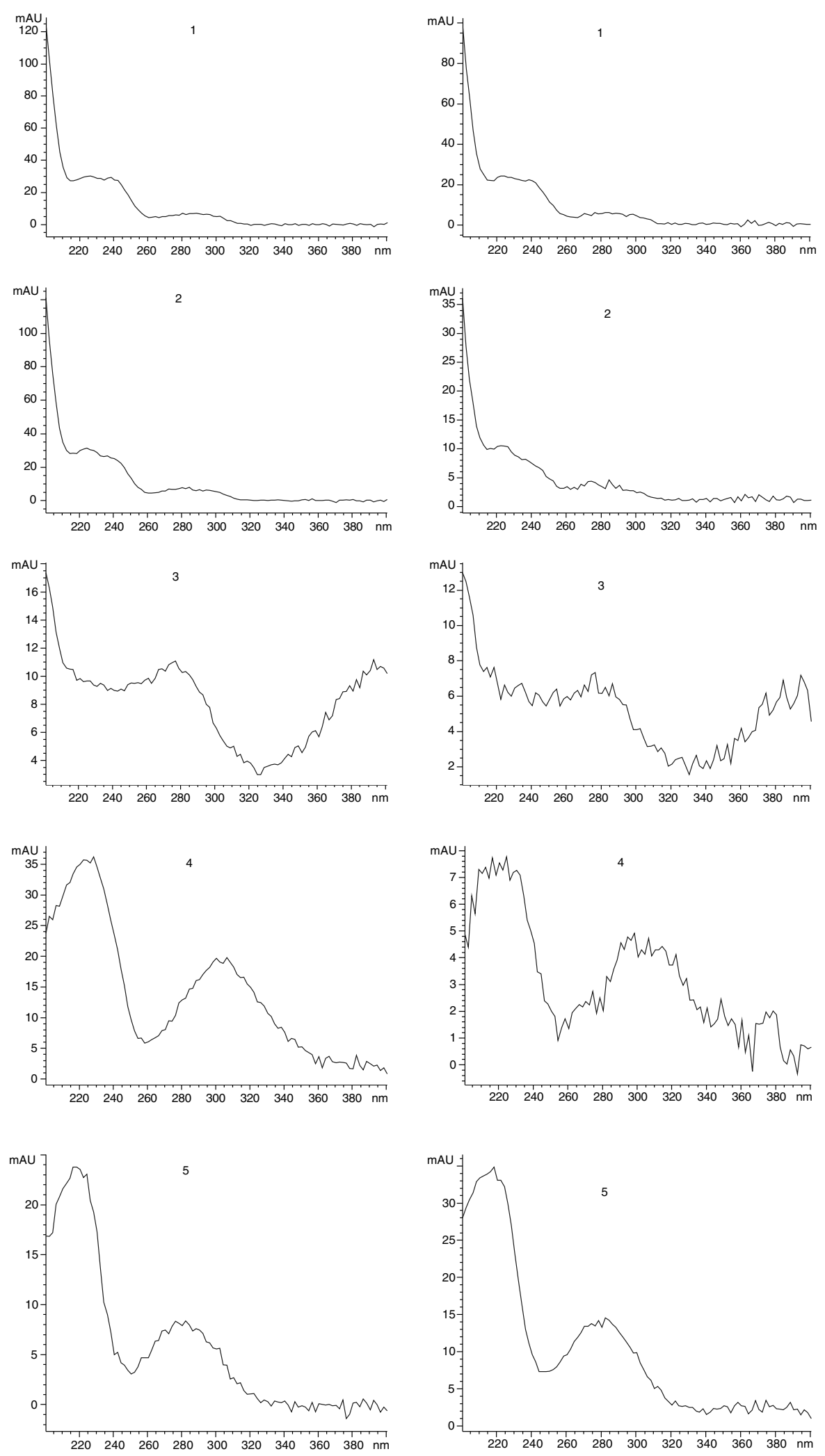

Fig. 9. UV spectra of five standard polar natural products (left) and on-line spectra of five standard polar natural products (right). (1) salidroside (2) tyrosol (3) rhodionin (4) ethyl gallate (5) gallic acid
Table 1. Relative standard deviation (RSD) of migration time and peak area

\begin{tabular}{|llr|}
\hline Analytes & $\begin{array}{l}\text { RSD } \\
\text { (migration time) }\end{array}$ & $\begin{array}{l}\text { RSD } \\
\text { (peak area) }\end{array}$ \\
\hline 1 & $0.25 \%$ & $5.26 \%$ \\
2 & $0.39 \%$ & $3.52 \%$ \\
3 & $0.44 \%$ & $21.03 \%$ \\
4 & $0.72 \%$ & $5.13 \%$ \\
5 & $1.41 \%$ & $17.27 \%$ \\
\hline
\end{tabular}

\section{Conclusion}

The work presented demonstrates that the five pharmacologically active compounds can be baseline separated. Complete baseline resolution with the proposed optimal conditions takes less than $7 \mathrm{~min}$ by using capillary electrophoresis. The method developed shows good retention time repeatability for the analysis of active compounds from extracted Rhodiola plants.

\section{Acknowledgements}

We would like to thank Ms Yan Cheng and $\mathrm{Mr}$ Yongfei Ming for their kindly technical assistance. This work was financed by grants from the "Wester Light" programme of talent cultivation of The Chinese Academy of Sciences (CAS) and Ministry of the Personnel of China and the National Science Foundation under Grant 20075016.

\section{References}

1. Alpert AJ (1990) J Chromatogr 499:177196

2. Churms SC (1995) J Chromatogr Libr 58:103-146

3. Alpert AJ, Shukla AK, Zieske LR, Yuen SW, Ferguson MAJ, Mehlert A, Pauly M, Orlando R (1994) J Chromatogr A 676:191-202

4. Guile GR, Rudd PM, Wing DR, Prime SB, Dwek RA (1996) Anal Biochem 240:210 226

5. Oyler AR, Armstrong BL, Cha JY, Zhou MX, Yang Q, Robinson RI, Dunphy R, Burinsky DJ (1996) J Chromatogr A 724:378-383

6. Zhu BY, Mant CT, Hodges RS (1991) J Chromatogr 548:13-24

7. Zhu BY, Mant CT, Hodges RS (1992) J Chromatogr 594:75-86

8. Issaq HJ (1999) Electrophoresis 20:31903202

9. Issaq HJ (1997) Electrophoresis 18:24382452

10. Larger PJ, Jones AD, Dacombe C (1998) J Chromatogr A 799:309-320 
11. Long H, Yang JJ, Liu HW, Wang TS (1999) 8:152-157

12. Bo T, Yang XD, Liu F, Li KA, Xiu LZ (2002) Analytica Chimica Acta 474:37-48

13. Liu SW (1999) Flora Qinghaiica, Qinghai People's Publishing House

14. Yin GS, Guo JQ, Wang GX (1992) Acta Nutrimenta sinica 20:98-102

15. Wang W, Guan GM, Jiang WH (1997) J. N. Bethune Univ Med Sci 23:20-22

16. Sun F, Sun H, Lu CM, Yang JX (1997) Chinese Pharmacological Bulletin 13:525528

17. Wang XQ, Sun F, Li JB (1993) Chinese Pharmacological Bulletin 9:443-445

18. Sergey N, Udintse V, Vladimir P, Chakov S (1991) 27:1182
Table 2. Quantitative equation and their liner range

\begin{tabular}{|llll|}
\hline Analytes & $\begin{array}{l}\text { Linear } \\
\text { regression }\end{array}$ & $\begin{array}{l}\text { Linear range } \\
\left(\mathrm{mmol} \mathrm{L}^{-1}\right)\end{array}$ & $\begin{array}{l}\text { Regression } \\
\text { coefficents }\end{array}$ \\
\hline 1 & $\mathrm{y}=1.45435+73.68696 \mathrm{x}$ & $0.025 \sim 0.2$ & 0.9954 \\
2 & $\mathrm{y}=0.53261+70.93217 \mathrm{x}$ & $0.025 \sim 0.2$ & 0.9988 \\
3 & $\mathrm{y}=-0.25+144.14286 \mathrm{x}$ & $0.05 \sim 0.2$ & 0.9890 \\
4 & $\mathrm{y}=3.45+144.42857 \mathrm{x}$ & $0.05 \sim 0.2$ & 0.9805 \\
5 & $\mathrm{y}=0.67391+279.47826 \mathrm{x}$ & $0.025 \sim 0.2$ & 0.9998 \\
\hline
\end{tabular}

19. Yu WS, Chen XM, Yang L, Li H, Li YF (1992) Natural Product R \& D. 4:23-28

20. Wang S, Wang FP (1992) Acta Pharmaceutica Sinica 27:117-120

21. Wang S, Wang FP (1991) Natural Product R \& D. 4:58-65
22. Colon LA, Reynolds KJ, Maldonado RA, Fermier AM (1997) Electrophoresis 18:2162

23. Dittmann M, Rozing G (1997) J.Microcol 9:399-406 